\title{
Identifying regulatory elements and gene boundaries with comparative genomic sequence analysis Jim Lund
}

Address: Department of Biology, University of Kentucky, Lexington, KY 40506, USA

Email: Jim Lund - jiml@uky.edu

from UT-ORNL-KBRIN Bioinformatics Summit 2008

Cadiz, KY, USA. 28-30 March 2008

Published: 8 July 2008

BMC Bioinformatics 2008, 9(Suppl 7):PI7 doi:I0.II86/I47|-2105-9-S7-PI7

This abstract is available from: http://www.biomedcentral.com/I47I-2/05/9/S7/PI7

(c) 2008 Lund; licensee BioMed Central Ltd.

For several groups of organisms multiple genomes have been sequenced either completely or to the unfinished shotgun sequencing stage. Comparative sequence analysis can identify functional sequence elements for pairs of organisms that diverged far enough in the past to allow mutational drift of non-conserved sequences. While analysis of a pair of genomes identifies many of these functional elements adding additional genomes allows additional information to be elicited.

Additional conserved sequence elements are identified as additional genomes are analyzed. These conserved sequence elements are often regulatory elements although they are difficult to classify with in silico analysis. For an individual gene, the set of associated conserved sequence elements and the organisms they are found in provides insight into the evolutionary history of the regulation of the gene.

The eight complete and unfinished shotgun sequenced nematode genomes and the dozen informative insect genome sequences were used to analyze conserved noncoding sequences in these groups of organisms. I have developed web-based software to allow researchers to explore and visualize sequence conservation that differs from previous work in analyzing conserved sequences in each pair of organisms rather than with respect to a single reference genome.

We have used this analysis to identify gene regulatory boundaries in the nematode C. elegans. The genomes of $C$. elegans and other nematodes have diverged enough that synteny regions are typically one or two genes long. I was able to associate conserved sequence elements to particular genes identifying the natural boundaries between genes and the extent of worm promoters.

\section{Acknowledgements}

This work was supported by Grant Number P20RR-1648I from the National Center for Research Resources (NCRR), a component of the National Institute of Health (NIH). 